\title{
Asymptotic Behavior of Solutions to a Vector Integral Equation with Deviating Arguments
}

\author{
Cristóbal González and Antonio Jiménez-Melado \\ Departamento de Análisis Matemático, Facultad de Ciencias, Universidad de Málaga, 29071 Málaga, Spain \\ Correspondence should be addressed to Cristóbal González; cmge@uma.es
}

Received 31 July 2013; Accepted 5 November 2013

Academic Editor: István Györi

Copyright @ 2013 C. González and A. Jiménez-Melado. This is an open access article distributed under the Creative Commons Attribution License, which permits unrestricted use, distribution, and reproduction in any medium, provided the original work is properly cited.

\begin{abstract}
In this paper, we propose the study of an integral equation, with deviating arguments, of the type $y(t)=\omega(t)-$ $\int_{0}^{\infty} f\left(t, s, y\left(\gamma_{1}(s)\right), \ldots, y\left(\gamma_{N}(s)\right)\right) d s, t \geq 0$, in the context of Banach spaces, with the intention of giving sufficient conditions that ensure the existence of solutions with the same asymptotic behavior at $\infty$ as $\omega(t)$. A similar equation, but requiring a little less restrictive hypotheses, is $y(t)=\omega(t)-\int_{0}^{\infty} q(t, s) F\left(s, y\left(\gamma_{1}(s)\right), \ldots, y\left(\gamma_{N}(s)\right)\right) d s, t \geq 0$. In the case of $q(t, s)=(t-s)_{+}$, its solutions with asymptotic behavior given by $\omega(t)$ yield solutions of the second order nonlinear abstract differential equation $y^{\prime \prime}(t)-\omega^{\prime \prime}(t)+F\left(t, y\left(\gamma_{1}(t)\right), \ldots, y\left(\gamma_{N}(t)\right)\right)=0$, with the same asymptotic behavior at $\infty$ as $\omega(t)$.
\end{abstract}

\section{Introduction}

From the pioneering work of Atkinson [1], and subsequent works found in the literature (see, e.g., [2-10] for recent papers on the subject), we consider the following differential problem, with deviating arguments:

$y^{\prime \prime}(t)-\omega^{\prime \prime}(t)+F\left(t, y\left(\gamma_{1}(t)\right), \ldots, y\left(\gamma_{N}(t)\right)\right)=0, \quad t \geq 0$,

with the task of finding solutions $y$ with the same behavior at $\infty$ as $\omega$. Solutions with this prescription are given by the solutions of the following integral equation:

$$
y(t)=\omega(t)-\int_{t}^{\infty}(s-t) F\left(s, y\left(\gamma_{1}(s)\right), \ldots, y\left(\gamma_{N}(s)\right)\right) d s,
$$

which, by writing $f\left(t, s, y\left(\gamma_{1}(s)\right), \ldots, y\left(\gamma_{N}(s)\right)\right)=(s-$ $t)_{+} F\left(s, y\left(\gamma_{1}(s)\right), \ldots, y\left(\gamma_{N}(s)\right)\right)$, is of the type

$$
y(t)=\omega(t)-\int_{0}^{\infty} f\left(t, s, y\left(\gamma_{1}(s)\right), \ldots, y\left(\gamma_{N}(s)\right)\right) d s,
$$

The purpose of this note is to provide conditions that ensure the existence of solutions to the above integral equation, whose asymptotic behavior at $\infty$ is the same as that of $\omega$, thus giving a procedure to show existence of solutions with prescribed asymptotic behavior of differential equation of the type (1). Our wish is to also work out this integral equation in the setting of Banach spaces.

Denote by $\mathbb{R}^{+}$the set $[0, \infty)$ of nonnegative real numbers. Assume that $\left\{\gamma_{j}\right\}_{j=1}^{N}$ is a finite set of continuous mappings from $\mathbb{R}^{+}$to $\mathbb{R}^{+}$, that $\left(X,\|\cdot\|_{X}\right)$ is a Banach space (with norm $\|\cdot\|_{X}$ ), and also that $\omega$ is a continuous mapping from $\mathbb{R}^{+}$to $X$. Finally, assume that $f: \mathbb{R}^{+} \times \mathbb{R}^{+} \times X^{N} \rightarrow X$ is a given continuous mapping with certain regularity and integrability conditions, to be specified later. In order to give a better aspect to our equation, define, for each continuous $y: \mathbb{R}^{+} \rightarrow X$, the mapping $\Gamma(y): \mathbb{R}^{+} \rightarrow X^{N}$ given by

$$
\Gamma(y)(t)=\left(y\left(\gamma_{1}(t)\right), \ldots, y\left(\gamma_{N}(t)\right)\right), \quad t \geq 0 .
$$

Then, our equation becomes

$$
y(t)=\omega(t)-\int_{0}^{\infty} f(t, s, \Gamma(y)(s)) d s, \quad t \geq 0,
$$


which, by writing $x(t)=y(t)-\omega(t), t \in \mathbb{R}^{+}$, is transformed into

$$
x(t)=-\int_{0}^{\infty} f(t, s, \Gamma(\omega+x)(s)) d s, \quad t \geq 0 .
$$

A bit more of notation and preliminary results are needed. As customary, $B_{X}(x, r)$ denotes the open ball in $X$ centered at $x$ with radius $r$. The closure in $X$ of any set $A \subseteq X$ is written $\bar{A}$, and its closed convex hull, $\overline{\mathrm{co}}(A)$. The space of continuous $X$-valued functions defined on $\mathbb{R}^{+}$is denoted by $\mathscr{C}\left(\mathbb{R}^{+}, X\right)$, while the space of bounded ones is $\mathscr{C}_{b}\left(\mathbb{R}^{+}, X\right)$. The latter is a Banach space when endowed with the sup norm $\|\cdot\|_{\infty}$, (i.e., for $\left.x \in \mathscr{C}_{b}\left(\mathbb{R}^{+}, X\right),\|x\|_{\infty}=\sup _{t \in \mathbb{R}^{+}}\|x(t)\|_{X}\right)$.

The Schauder fixed point theorem states that any continuous operator $T$ defined on a nonempty, bounded, closed and convex subset $C$ of a Banach space has necessarily a fixed point, provided that $T(C)$ is a relatively compact subset of $C$. We will also be needing a well-known version of the Arzelà-Ascoli theorem which, in the case that occupies us, is as follows: if a family $\mathscr{F} \subseteq \mathscr{C}\left(\mathbb{R}^{+}, X\right)$ is equicontinuous at each $t \in \mathbb{R}^{+}$, and each section $\mathscr{F}(t):=\{u(t): u \in \mathscr{F}\}$ is relatively compact in $X$, then each sequence $\left\{u_{n}\right\} \subseteq \mathscr{F}$ contains a subsequence that converges uniformly on compact subsets of $\mathbb{R}^{+}$to a given $X$-valued function $u$.

Let us also say a word about vector integrals. For a brief introduction see, for example, [11] or [12]. If $f:[a, b] \rightarrow X$ is a bounded vector function, the Riemann sum of $f$ associated to a finite partition of $[a, b], \wp=\left\{a=t_{0}<t_{1}<\cdots<t_{n}=b\right\}$ (with norm $\|\wp\|=\max _{j}\left|t_{j}-t_{j-1}\right|$ ), and to a selection $\left\{t_{j}^{*}\right\}_{j=1}^{n}$, $t_{j}^{*} \in\left[t_{j-1}, t_{j}\right]$, is $\sum_{j=1}^{n} f\left(t_{j}^{*}\right)\left(t_{j}-t_{j-1}\right)$. Without specifying the type of limit alluded, we say that $f$ is Riemann integrable on $[a, b]$ if there exists the limit of its Riemann sums as the norm of the partitions of $[a, b]$ tends to 0 , in which case, this limit (which is unique) is called the integral of $f$ over $[a, b]$ and is denoted by $\int_{a}^{b} f(t) d t$. That is, being a little bit sloppy with the notation, we have

$$
\int_{a}^{b} f(t) d t=\lim _{\|\wp\| \rightarrow 0} \sum_{j} f\left(t_{j}^{*}\right)\left(t_{j}-t_{j-1}\right) .
$$

Observe that whenever $f$ is integrable on $[a, b]$, then each Riemann sum associated to $f$ is $(b-a)$ times a linear convex combination of elements of $f([a, b])$. Therefore, the integral of $f$ over $[a, b]$, being a limit of Riemann sums, is a multiple of an element of the closed convex hull of $f([a, b])$, that is,

$$
\begin{aligned}
\int_{a}^{b} f(t) d t= & \lim _{\|\wp\| \rightarrow 0}(b-a) \\
& \times \sum_{j} f\left(t_{j}^{*}\right) \frac{t_{j}-t_{j-1}}{b-a} \in(b-a) \overline{\operatorname{co}} f([a, b]) .
\end{aligned}
$$

\section{Existence of Solutions}

We begin this section enumerating the conditions that will be basic for our results on existence of solutions of $(E)$. Take $f, \gamma_{j}$, and $\omega$ as above and, in analogy with what would happen with continuous mappings on finite dimensional spaces, assume that

$f$ is uniformly continuous on bounded sets

of $\mathbb{R}^{+} \times \mathbb{R}^{+} \times X^{N}$, and maps bounded

sets into relatively compact sets of $X$.

The following conditions have already been motivated in previous work [7]. Recall that $\omega: \mathbb{R}^{+} \rightarrow X$ is continuous, but not necessarily bounded.

There exists $g: \mathbb{R}^{+} \longrightarrow \mathbb{R}^{+}$bounded, with $g(t) \stackrel{t \rightarrow \infty}{\longrightarrow} 0$, such that for $C=\left\{y \in \mathscr{C}\left(\mathbb{R}^{+}, X\right)\right.$ :

$$
\begin{array}{r}
\left.\|y(t)-\omega(t)\|_{X} \leq g(t), t \in \mathbb{R}^{+}\right\}, \\
\int_{0}^{\infty}\|f(t, s, \Gamma(y)(s))\|_{X} d s \leq g(t), \quad \forall t \in \mathbb{R}^{+}, \forall y \in C .
\end{array}
$$

There exists $h: \mathbb{R}^{+} \longrightarrow \mathbb{R}^{+}$with $h(\tau) \stackrel{\tau \longrightarrow \infty}{\longrightarrow} 0$, such that

$$
\int_{\tau}^{\infty}\|f(t, s, \Gamma(y)(s))\|_{X} d s \leq h(\tau), \quad \forall t, \tau \in \mathbb{R}^{+}, \forall y \in C .
$$

The result on existence of solutions to the integral equation is the following.

Theorem 1. Under hypotheses (H0), (H1), and (H2), the integral equation $(E)$ has a solution $y(t)$ asymptotically equal to $\omega(t)$ as $t \rightarrow \infty$.

Remark 2. This theorem represents a generalization of the one presented in the work [7] in two aspects. First, we have made the jump to deal with integral equations in the setting of infinite dimensional spaces. And second, we have included deviating arguments in the equation.

Proof of Theorem 1. First observe that it suffices to find a solution to the integral equation $(\widetilde{E})$ in $\widetilde{C}=\left\{x \in \mathscr{C}_{b}\left(\mathbb{R}^{+}, X\right)\right.$ : $\left.\|x(t)\|_{X} \leq g(t), t \in \mathbb{R}^{+}\right\}$, and this will be achieved by proving the existence of a fixed point in $\widetilde{C}$ of the operator:

$$
\begin{gathered}
T: x \in \widetilde{C} \longmapsto T x, \\
T x(t)=-\int_{0}^{\infty} f(t, s, \Gamma(\omega+x)(s)) d s, \quad t \geq 0 .
\end{gathered}
$$

We proceed to check that the conditions of the Schauder fixed point Theorem are fulfilled. First observe that $\widetilde{C}$ is a nonempty, bounded, closed, and convex subset of the Banach space $\mathscr{C}_{b}\left(\mathbb{R}^{+}, X\right)$. Also, by $(\mathrm{H} 1),\|T x(t)\|_{X} \leq g(t)$ for all $t \in \mathbb{R}^{+}$and all $x \in \widetilde{C}$. So $T \widetilde{C} \subseteq \widetilde{C}$ provided $T x \in \mathscr{C}\left(\mathbb{R}^{+}, X\right)$ for all $x \in \widetilde{C}$.

In fact, we will prove four assertions. (a) $T \widetilde{C}$ is uniformly equicontinuous on $\mathbb{R}^{+}$. This will give the desired continuity 
of $T x$ for each $x \in \widetilde{C}$. (b) $T$ is uniformly continuous on $\widetilde{C}$, hence it will be continuous on $\widetilde{C}$. (c) $T \widetilde{C}\left(\mathbb{R}^{+}\right)$is relatively compact in $X$. Thus, (a) and (c) will imply, by the ArzelàAscoli theorem, that each sequence in $T \widetilde{C}$ has a subsequence which converges uniformly on each compact subset of $\mathbb{R}^{+}$to a given function in $\mathscr{C}\left(\mathbb{R}^{+}, X\right)$ (actually in $\widetilde{C}$ ). Finally, in order to obtain that $T \widetilde{C}$ is relatively compact in $\mathscr{C}_{b}\left(\mathbb{R}^{+}, X\right)$, we will use the "funnel" structure of $\widetilde{C}$ to prove (d), that any sequence in $\widetilde{C}$ which converges uniformly on each compact subset of $\mathbb{R}^{+}$to a given function in $\widetilde{C}$ must indeed converge uniformly to that function in all of $\mathbb{R}^{+}$. With all these assertions, the Schauder fixed point theorem can be applied to conclude the existence of a fixed point of $T$, as we want.

Start fixing an arbitrary $\varepsilon>0$ once for all. In what follows, we will build up different objects indexed by this $\varepsilon,\left(t_{\varepsilon}, \tau_{\varepsilon}, D_{\varepsilon}\right.$, $\left.B_{\varepsilon}\right)$, knowing that even if for each assertion we have to start taking an arbitrary $\varepsilon>0$, the objects will vary accordingly but not the way to obtain them.

Since $g(t) \rightarrow 0$ as $t \rightarrow \infty$, there exists $t_{\varepsilon}>0$ such that

$$
g(t)<\frac{\varepsilon}{2}, \quad \forall t \geq t_{\varepsilon}
$$

We start proving (d), as it is quite independent of the rest of assertions. Assume that a sequence $\left\{u_{n}\right\} \subseteq \widetilde{C}$ converges uniformly on each compact subset of $\mathbb{R}^{+}$to a function $u \in$ $\widetilde{C}$, and let us show that indeed converges uniformly to $u$ in all of $\mathbb{R}^{+}$. For the $\varepsilon>0$ above (so for any $\varepsilon>0$ ), find the corresponding $t_{\varepsilon}>0$ to satisfy (8). Since $u_{n}, n \in \mathbb{N}$, and $u$ all belong to $\widetilde{C}$, then

$$
\left\|u_{n}(t)-u(t)\right\|_{X} \leq 2 g(t), \quad \forall n \in \mathbb{N}, \forall t \in \mathbb{R}^{+} .
$$

In particular,

$$
\left\|u_{n}(t)-u(t)\right\|_{X} \leq 2 g(t)<\varepsilon, \quad \forall n \in \mathbb{N}, \forall t \geq t_{\varepsilon} .
$$

Now, since $\left\{u_{n}\right\}$ converges uniformly to $u$ in $\left[0, t_{\varepsilon}\right]$, there exists $n_{\varepsilon} \in \mathbb{N}$ such that

$$
\left\|u_{n}(t)-u(t)\right\|_{X}<\varepsilon, \quad \forall n \geq n_{\varepsilon}, \forall t \in\left[0, t_{\varepsilon}\right] .
$$

This tells us that if $n \geq n_{\varepsilon}$, then $\left\|u_{n}-u\right\|_{\infty} \leq \varepsilon$, proving that the convergence of $\left\{u_{n}\right\}$ to $u$ is uniform on $\mathbb{R}^{+}$, and thus (d) is proven.

Next, continue building up other objects associated with the arbitrary $\varepsilon$ fixed above. Observe that, by (8) and (H1),

$$
\begin{aligned}
\|T x(t)\|_{X} & \leq \int_{0}^{\infty}\|f(t, s, \Gamma(\omega+x)(s))\|_{X} d s \\
& \leq g(t)<\frac{\varepsilon}{2}, \quad \forall t \geq t_{\varepsilon}, \quad \forall x \in \widetilde{C} .
\end{aligned}
$$
that

Also, since $h(\tau) \rightarrow 0$ as $\tau \rightarrow \infty$, there exists $\tau_{\varepsilon}>0$ such

$$
h(\tau)<\frac{\varepsilon}{4}, \quad \forall \tau \geq \tau_{\varepsilon}
$$

so, by (H2),

$$
\begin{aligned}
& \int_{\tau_{\varepsilon}}^{\infty}\|f(t, s, \Gamma(\omega+x)(s))\|_{X} d s \\
& \quad \leq h\left(\tau_{\varepsilon}\right)<\frac{\varepsilon}{4}, \quad \forall t \in \mathbb{R}^{+}, \forall x \in \widetilde{C} .
\end{aligned}
$$

The continuity of $\omega$ and the $\gamma_{j}$ 's, $j=1, \ldots, N$, and the uniform bound for functions in $\widetilde{C}$ (given by the bound of $g$ ) imply that there exists a bounded set $D_{\varepsilon} \subseteq X^{N}$, depending on $\tau_{\varepsilon}, \omega$, the $\gamma_{j}$ 's, and $g$, but not on $x \in \widetilde{C}$, such that

$$
\begin{array}{r}
\Gamma(\omega+x)(s) \\
=\left(\omega\left(\gamma_{1}(s)\right)+x\left(\gamma_{1}(s)\right), \ldots, \omega\left(\gamma_{N}(s)\right)+x\left(\gamma_{N}(s)\right)\right) \in D_{\varepsilon}, \\
\forall x \in \widetilde{C}, \forall s \in\left[0, \tau_{\varepsilon}\right] .
\end{array}
$$

Now, observe that $B_{\varepsilon}:=\left[0, t_{\varepsilon}+1\right] \times\left[0, \tau_{\varepsilon}\right] \times D_{\varepsilon}$ is bounded in $\mathbb{R}^{+} \times \mathbb{R}^{+} \times X^{N}$, so by (H0),

$f$ is uniformly continuous on $B_{\varepsilon}$,

and $f\left(B_{\varepsilon}\right)$ is relatively compact in $X$.

By the uniform continuity of $f$ on $B_{\varepsilon}$, there exists $\delta_{\varepsilon} \epsilon$ $(0,1)$ such that, whenever $\left(t_{1}, s_{1}, y_{1,1}, \ldots, y_{N, 1}\right) \in B_{\varepsilon}$ and $\left(t_{2}, s_{2}, y_{1,2}, \ldots, y_{N, 2}\right) \in B_{\varepsilon}$ with $\left|t_{1}-t_{2}\right|<\delta_{\varepsilon},\left|s_{1}-s_{2}\right|<\delta_{\varepsilon}$, and $\left\|y_{j, 1}-y_{j, 2}\right\|_{X}<\delta_{\varepsilon}, j=1, \ldots, N$, then

$$
\left\|f\left(t_{1}, s_{1}, y_{1,1}, \ldots, y_{N, 1}\right)-f\left(t_{2}, s_{2}, y_{1,2}, \ldots, y_{N, 2}\right)\right\|_{X}<\frac{\varepsilon}{4 \tau_{\varepsilon}} \text {. }
$$

Notice that if $t_{1}, t_{2} \in \mathbb{R}^{+}$with $\left|t_{1}-t_{2}\right|<\delta_{\varepsilon}$ and, without loss of generality, $t_{1} \leq t_{2}$, then several cases are possible. If $t_{2} \geq t_{\varepsilon}+1$, then, as $\delta_{\varepsilon}<1, t_{2} \geq t_{1}>t_{2}-\delta_{\varepsilon}>t_{\varepsilon}+1-1=t_{\varepsilon}$, and so, by (12),

$$
\begin{array}{r}
\left\|T x\left(t_{1}\right)-T x\left(t_{2}\right)\right\|_{X} \leq\left\|T x\left(t_{1}\right)\right\|_{X}+\left\|T x\left(t_{2}\right)\right\|_{X}<\varepsilon, \\
\forall x \in \widetilde{C} .
\end{array}
$$

If, on the other hand, $t_{2} \leq t_{\varepsilon}+1$, then, by (15), $\Gamma(\omega+$ $x)\left(\left[0, \tau_{\varepsilon}\right]\right) \subseteq D_{\varepsilon}$ for all $x \in \widetilde{C}$, and, by (17) and (14), we have, for any $x \in \widetilde{C}$,

$$
\begin{aligned}
& \left\|T x\left(t_{1}\right)-T x\left(t_{2}\right)\right\|_{X} \\
& \leq \int_{0}^{\tau_{\varepsilon}} \| f\left(t_{2}, s, \Gamma(\omega+x)(s)\right) \\
& \quad-f\left(t_{1}, s, \Gamma(\omega+x)(s)\right) \|_{X} d s \\
& \quad+\int_{\tau_{\varepsilon}}^{\infty}\left\|f\left(t_{1}, s, \Gamma(\omega+x)(s)\right)\right\|_{X} d s \\
& \quad+\int_{\tau_{\varepsilon}}^{\infty}\left\|f\left(t_{2}, s, \Gamma(\omega+x)(s)\right)\right\|_{X} d s \\
& <\tau_{\varepsilon} \frac{\varepsilon}{4 \tau_{\varepsilon}}+2 \frac{\varepsilon}{4}<\varepsilon .
\end{aligned}
$$

This proves (a), the uniform equicontinuity of $T \widetilde{C}$ over $\mathbb{R}^{+}$. 
Now notice that if $x_{1}, x_{2} \in \widetilde{C}$ with $\left\|x_{1}-x_{2}\right\|_{\infty}<\delta_{\varepsilon}$ and $t \in \mathbb{R}^{+}$, then, again, two cases are possible. If $t \geq t_{\varepsilon}$, then, by (12),

$$
\left\|T x_{1}(t)-T x_{2}(t)\right\|_{X} \leq\left\|T x_{1}(t)\right\|_{X}+\left\|T x_{2}(t)\right\|_{X} \leq 2 g(t)<\varepsilon,
$$

while for $t \leq t_{\varepsilon}$, using (15), (17), (14), and (13),

$$
\begin{aligned}
& \left\|T x_{1}(t)-T x_{2}(t)\right\|_{X} \\
& \leq \int_{0}^{\tau_{\varepsilon}} \| f\left(t, s, \Gamma\left(\omega+x_{2}\right)(s)\right) \\
& \quad-f\left(t, s, \Gamma\left(\omega+x_{1}\right)(s)\right) \|_{X} d s \\
& \quad+\int_{\tau_{\varepsilon}}^{\infty}\left\|f\left(t, s, \Gamma\left(\omega+x_{1}\right)(s)\right)\right\|_{X} d s \\
& \quad+\int_{\tau_{\varepsilon}}^{\infty}\left\|f\left(t, s, \Gamma\left(\omega+x_{2}\right)(s)\right)\right\|_{X} d s \\
& <\tau_{\varepsilon} \frac{\varepsilon}{4 \tau_{\varepsilon}}+2 h\left(\tau_{\varepsilon}\right)<\varepsilon,
\end{aligned}
$$

This proves that $\left\|T x_{1}-T x_{2}\right\|_{\infty}<\varepsilon$, showing (b), that $T$ is uniformly continuous on $\widetilde{C}$.

For the compactness of $\overline{T \widetilde{C}\left(\mathbb{R}^{+}\right)}$in $X$, it suffices to show that $T \widetilde{C}\left(\mathbb{R}^{+}\right)$is totally bounded [13, page 298]; that is, for the given $\varepsilon>0$ (so for any $\varepsilon>0$ ) there exists a finite covering of $T \widetilde{C}\left(\mathbb{R}^{+}\right)$with balls of radii not bigger than $\varepsilon$. Observe first that, by (12), $\|T x(t)\|_{X}<\varepsilon / 2$ for all $x \in \widetilde{C}$ and all $t \geq t_{\varepsilon}$, that is,

$$
T \widetilde{C}\left(\left[t_{\varepsilon}, \infty\right)\right) \subseteq B_{X}\left(0, \frac{\varepsilon}{2}\right) .
$$

Now, in order to control the elements of $T \widetilde{C}\left(\left[0, t_{\varepsilon}\right]\right)$, observe that each of these can be decomposed as the sum of a "head" and a "tail,"

$$
\begin{array}{r}
T x(t)=-\int_{0}^{\tau_{\varepsilon}} f(t, s, \Gamma(\omega+x)(s)) d s \\
-\int_{\tau_{\varepsilon}}^{\infty} f(t, s, \Gamma(\omega+x)(s)) d s, \\
\forall x \in \widetilde{C} \text { and all } t \in\left[0, t_{\varepsilon}\right] .
\end{array}
$$

The "head" can be approximated by Riemann sums, which, in turn, are nothing else but $\tau_{\varepsilon}$ times a convex linear combination of elements of $-f\left(B_{\varepsilon}\right)$, that is, the "head" is an element of $\tau_{\varepsilon} \overline{\mathrm{co}}\left(-f\left(B_{\varepsilon}\right)\right)$. By $(16), f\left(B_{\varepsilon}\right)$ has compact closure in $X$, so by Mazur's theorem [14], $\overline{\mathrm{co}}\left(-f\left(B_{\varepsilon}\right)\right)$ is compact, and therefore it can be covered with a finite number of balls, say $B_{1}, \ldots, B_{\ell}$, of radii not bigger than $\varepsilon /\left(2 \tau_{\varepsilon}\right)$. This yields a finite covering of $\tau_{\varepsilon} \overline{\mathrm{cO}}\left(-f\left(B_{\varepsilon}\right)\right)$ with balls of radii not bigger than $\varepsilon / 2$, precisely the collection $\left\{\tau_{\varepsilon} B_{j}\right\}_{j=1}^{\ell}$. On the other hand, by (14), the "tail" of each of the above integrals is bounded by $h\left(\tau_{\varepsilon}\right)<\varepsilon / 4$, so they are elements of $B_{X}(0, \varepsilon / 4)$. All this can be summarized as follows:

$$
\begin{aligned}
T \widetilde{C}\left(\left[0, t_{\varepsilon}\right]\right) & \subseteq \tau_{\varepsilon} \overline{\operatorname{co}}\left(-f\left(B_{\varepsilon}\right)\right)+B_{X}\left(0, \frac{\varepsilon}{4}\right) \\
& \subseteq\left(\bigcup_{j=1}^{\ell} \tau_{\varepsilon} B_{j}\right)+B_{X}\left(0, \frac{\varepsilon}{4}\right) \\
& =\bigcup_{j=1}^{\ell}\left(\tau_{\varepsilon} B_{j}+B_{X}\left(0, \frac{\varepsilon}{4}\right)\right) ;
\end{aligned}
$$

that is, $T \widetilde{C}\left(\left[0, t_{\varepsilon}\right]\right)$ can be covered with a finite collection of balls of radii smaller than $\varepsilon$, because each $\tau_{\varepsilon} B_{j}+B_{X}(0, \varepsilon / 4)$ is readily seen to be a ball of radius not bigger than $3 \varepsilon / 4$.

At the end, by (22) and (24), we have

$$
\begin{aligned}
T \widetilde{C}\left(\mathbb{R}^{+}\right) & \subseteq T \widetilde{C}\left(\left[0, t_{\varepsilon}\right]\right) \cup T \widetilde{C}\left(\left[t_{\varepsilon}, \infty\right)\right) \\
& \subseteq \bigcup_{j=1}^{\ell}\left(\tau_{\varepsilon} B_{j}+B_{X}\left(0, \frac{\varepsilon}{4}\right)\right) \cup B_{X}\left(0, \frac{\varepsilon}{2}\right) ;
\end{aligned}
$$

that is, we have given a finite covering of $T \widetilde{C}\left(\mathbb{R}^{+}\right)$with balls of radii not bigger than $\varepsilon$. With this we conclude (c) and, with all four assertions proved, the theorem too.

Coming back to the integral equation (2) underlying the differential equation (1), we just need to adapt the hypotheses presented above to the function $f\left(t, s, y_{1}, \ldots, y_{N}\right)=$ $(s-t)_{+} F\left(s, y_{1}, \ldots, y_{N}\right)$, to obtain a corresponding result on existence of solutions to (2) with asymptotic behavior given by $\omega(t)$. Notice that these hypotheses, (H0), (H1), and (H2), are natural generalizations from the 1-dimensional case. However, the proof in the abstract setting has shown many more properties for the operator $T$ that needed in order to show the existence of a fixed point. This, somehow, is telling us that the hypotheses could be weakened. For the moment, we content ourselves noticing that, in (2), the corresponding hypothesis of uniform continuity on bounded sets is "not needed," because it will be "consequence" of the other hypotheses, and a little trick of changing the domain of definition of the operator. Thus, for our next result, we will be using the following hypothesis:

$$
F: \mathbb{R}^{+} \times X^{N} \longrightarrow X \text { is continuous and }
$$

maps bounded sets into relatively compact ones.

Also, instead of considering just the integral equation (2), we generalize a little bit to a convolution type integral equation,

$$
y(t)=\omega(t)-\int_{0}^{\infty} q(t, s) \quad F(s, \Gamma(y)(s)) d s, \quad t \geq 0
$$


with a kernel $q(t, s)$ satisfying the right properties for us. Observe that, when $q(t, s)=(s-t)_{+}$, and $0 \leq t_{1} \leq t_{2}$,

$$
\begin{aligned}
q\left(t_{1}, s\right)-q\left(t_{2}, s\right) & =\left(s-t_{1}\right)_{+}-\left(s-t_{2}\right)_{+} \\
& = \begin{cases}0 & \text { if } 0 \leq s \leq t_{1} \\
s-t_{1} & \text { if } t_{1} \leq s \leq t_{2} \\
t_{2}-t_{1} & \text { if } t_{2} \leq s\end{cases} \\
& \leq t_{2}-t_{1} .
\end{aligned}
$$

That is, $q(t, s)$ satisfies a Lipschitz condition on the first variable, independent of the second. Actually, much less is needed, just continuity of $q(t, s)$ suffices.

Theorem 3. Let $\omega, \gamma_{j}, j=1, \ldots, N, \Gamma$ be as for Theorem 1 , and let $q: \mathbb{R}^{+} \times \mathbb{R}^{+} \rightarrow \mathbb{R}$ be continuous. Under hypotheses $\left(H 0^{\prime}\right),(H 1)$, and $(H 2)$ (where $f\left(t, s, y_{1}, \ldots, y_{N}\right)=$ $\left.q(t, s) F\left(s, y_{1}, \ldots, y_{N}\right)\right)$, the integral equation $\left(E_{1}\right)$ has a solution $y(t)$ asymptotically equal to $\omega(t)$ as $t \rightarrow \infty$.

Remark 4. Observe, as in [7], that if the kernel $q$ is the one we started with, $q(t, s)=(s-t)_{+}$, then hypothesis $(\mathrm{H} 2)$ is redundant, because in that case, if $s \geq \tau$, we always have, for $\tau \leq t,(s-t)_{+} \leq(s-\tau)_{+} \leq 2(s-\tau / 2)_{+}$, and for $\tau>t$,

$$
(s-t)_{+}=s-t \leq s \leq 2\left(s-\frac{\tau}{2}\right)=2\left(s-\frac{\tau}{2}\right)_{+} .
$$

Consequently, from hypothesis (H1), taking $h(\tau)=3 g(\tau / 2)$, we have $h(\tau) \rightarrow 0$ as $\tau \rightarrow \infty$, and

$$
\begin{aligned}
& \int_{\tau}^{\infty}(s-t)_{+}\|F(s, \Gamma(y)(s))\|_{X} d s \\
& \quad \leq 3 g\left(\frac{\tau}{2}\right)=h(\tau), \quad \forall \tau, t \in \mathbb{R}^{+}, \forall y \in C .
\end{aligned}
$$

In general, hypothesis $(\mathrm{H} 2)$ is superfluous whenever there exist constants, $a \in(0,1)$ and $A>1$, such that

$$
|q(t, s)| \leq A|q(a \tau, s)|, \quad \forall t, s, \tau \in \mathbb{R}^{+} \text {, with } s \geq \tau .
$$

Proof of Theorem 3. We proceed as before. Consider initially the same nonempty, bounded, closed, and convex subset $\widetilde{C}$ as before, as well as the same operator $T$. The idea is to find a fixed point for $T$ using the Schauder fixed point theorem. For that, we start repeating the same scheme of four assertions established in the previous theorem. The two assertions that do not depend on the uniform continuity of $f$ on bounded sets are done the same way as before; hence we omit their proofs. These are (c), that $T \widetilde{C}\left(\mathbb{R}^{+}\right)$is compact in $X$, and (d), that uniform convergence on compact subsets of $\mathbb{R}^{+}$of a sequence in $\widetilde{C}$ turns into actual uniform convergence on $\mathbb{R}^{+}$.

Let us now prove (a), that $T \widetilde{C}$ is uniformly equicontinuous on $\mathbb{R}^{+}$. This will finish showing that $T \widetilde{C} \subseteq \widetilde{C}$, and, by (c), (d), and the Arzelà-Ascoli theorem, that $T \widetilde{C}$ has compact closure in $\mathscr{C}_{b}\left(R^{+}, X\right)$.

Let $\varepsilon>0$ be fixed. Find, as before, $t_{\varepsilon}>0, \tau_{\varepsilon}>0$, and $D_{\varepsilon}$ bounded subset of $X^{N}$, so as to satisfy (8), (12), (13), (14), and
(15). Now, observe that $\left[0, \tau_{\varepsilon}\right] \times D_{\varepsilon}$ is bounded in $\mathbb{R}^{+} \times X^{N}$, so by $\left(\mathrm{H0}^{\prime}\right), F\left(\left[0, \tau_{\varepsilon}\right] \times D_{\varepsilon}\right)$ is relatively compact in $X$; hence there exists $M_{\varepsilon}>0$ such that

$$
\|F(s, \Gamma(\omega+x)(s))\|_{X} \leq M_{\varepsilon}, \quad \forall s \in\left[0, \tau_{\varepsilon}\right], \forall x \in \widetilde{C} .
$$

Notice now that $q$ is uniformly continuous on $R_{\varepsilon}=\left[0, t_{\varepsilon}+\right.$ $1] \times\left[0, \tau_{\varepsilon}\right]$. So there is $\delta_{\varepsilon} \in(0,1)$ such that, whenever $\left(t_{1}, s_{1}\right)$ and $\left(t_{2}, s_{2}\right)$ are in $R_{\varepsilon}$ with $\left|t_{1}-t_{2}\right|<\delta$ and $\left|s_{1}-s_{2}\right|<\delta$, then

$$
\left|q\left(t_{1}, s_{1}\right)-q\left(t_{2}, s_{2}\right)\right|<\frac{\varepsilon}{4 \tau_{\varepsilon} M_{\varepsilon}}
$$

Now take $t_{1}, t_{2} \in \mathbb{R}^{+}$with $\left|t_{1}-t_{2}\right|<\delta_{\varepsilon}$ and, without loss of generality, assume that $t_{1} \leq t_{2}$. Then again, several cases are possible. If $t_{2} \geq t_{\varepsilon}+1$, then, as $\delta_{\varepsilon}<1, t_{2} \geq t_{1}>t_{2}-\delta_{\varepsilon}>$ $t_{\varepsilon}+1-1=t_{\varepsilon}$, and so, by (12),

$$
\begin{array}{r}
\left\|T x\left(t_{1}\right)-T x\left(t_{2}\right)\right\|_{X} \leq\left\|T x\left(t_{1}\right)\right\|_{X}+\left\|T x\left(t_{2}\right)\right\|_{X}<\varepsilon, \\
\forall x \in \widetilde{C} .
\end{array}
$$

If, on the other hand, $t_{2} \leq t_{\varepsilon}+1$, then, by (31), (30), and (14), we have, for any $x \in \widetilde{C}$,

$$
\begin{aligned}
& \left\|T x\left(t_{1}\right)-T x\left(t_{2}\right)\right\|_{X} \\
& \leq \int_{0}^{\tau_{\varepsilon}}\left|q\left(t_{2}, s\right)-q\left(t_{1}, s\right)\right| \\
& \quad \times\|F(s, \Gamma(\omega+x)(s))\|_{X} d s \\
& \quad+\int_{\tau_{\varepsilon}}^{\infty}\left|q\left(t_{1}, s\right)\right|\|F(s, \Gamma(\omega+x)(s))\|_{X} d s \\
& \quad+\int_{\tau_{\varepsilon}}^{\infty}\left|q\left(t_{2}, s\right)\right|\|F(s, \Gamma(\omega+x)(s))\|_{X} d s \\
& <\frac{\varepsilon}{4 \tau_{\varepsilon} M_{\varepsilon}} M_{\varepsilon} \tau_{\varepsilon}+2 \frac{\varepsilon}{4}<\varepsilon .
\end{aligned}
$$

This proves the uniform equicontinuity of $T \widetilde{C}$ over $\mathbb{R}^{+}$.

To finish the proof, we have to prove (b), that $T$ is uniformly continuous. It is here that we restrict the domain of definition of $T$. Observe that $\overline{\mathrm{CO}}(T \widetilde{C})$ is nonempty, closed and convex. Also, $\overline{\operatorname{co}}(T \widetilde{C}) \subseteq \widetilde{C}$ because $T \widetilde{C} \subseteq \widetilde{C}$ and $\widetilde{C}$ is closed and convex. More is true, since $T \widetilde{C}$ has compact closure, then, by Mazur's Theorem, $\overline{\mathrm{co}}(T \widetilde{C})$ is compact too. One more thing, $T$ leaves invariant $\overline{\mathrm{co}}(T \widetilde{\mathrm{C}})$ :

$$
\overline{\mathrm{co}}(T \widetilde{C}) \subseteq \widetilde{C} \Longrightarrow T(\overline{\mathrm{co}}(T \widetilde{C})) \subseteq T \widetilde{C} \subseteq \overline{\mathrm{co}}(T \widetilde{C})
$$

With all of this, it suffices to prove that $T$ is continuous on this new $T$-invariant set. Let us prove that, indeed, $T$ is uniformly continuous on $\overline{\mathrm{co}}(T \widetilde{C})$. Let $\varepsilon>0$ be given. Find that $t_{\varepsilon}>0$ and $\tau_{\varepsilon}>0$ as before, so as to satisfy (8), (12), (13), and (14). The continuity of $\omega$ and the $\gamma_{j}$ 's, $j=1, \ldots, N$, and the compactness of $\overline{\mathrm{co}}(T \widetilde{C})$ tells us that $D_{\varepsilon}=\left\{\Gamma(\omega+x)(s): s \in\left[0, \tau_{\varepsilon}\right], x \in \overline{\operatorname{co}}(T \widetilde{C})\right\}$ is a compact subset of $X^{N}$, giving us the opportunity to say that 
$F$ is uniformly continuous on $\left[0, \tau_{\varepsilon}\right] \times D_{\varepsilon}$ and, consequently, that $f\left(t, s, y_{1}, \ldots, y_{N}\right)=q(t, s) F\left(s, y_{1}, \ldots, y_{N}\right)$ is uniformly continuous on $B_{\varepsilon}:=\left[0, t_{\varepsilon}+1\right] \times\left[0, \tau_{\varepsilon}\right] \times D_{\varepsilon}$. So there exists $\delta_{\varepsilon} \in(0,1)$ such that, for $\left(t_{1}, s_{1}, y_{1,1}, \ldots, y_{N, 1}\right) \in B_{\varepsilon}$ and $\left(t_{2}, s_{2}, y_{1,2}, \ldots, y_{N, 2}\right) \in B_{\varepsilon}$ with $\left|t_{1}-t_{2}\right|<\delta_{\varepsilon},\left|s_{1}-s_{2}\right|<\delta_{\varepsilon}$, and $\left\|y_{j, 1}-y_{j, 2}\right\|_{X}<\delta_{\varepsilon}, j=1, \ldots, N$, then

$$
\left\|f\left(t_{1}, s_{1}, y_{1,1}, \ldots, y_{N, 1}\right)-f\left(t_{2}, s_{2}, y_{1,2}, \ldots, y_{N, 2}\right)\right\|_{X}<\frac{\varepsilon}{4 \tau_{\varepsilon}} .
$$

Now, it is a matter of repeating the same steps as was done in Theorem 1 to prove the uniform continuity of $T$.

This concludes the proof of the theorem.

Remark 5. Instead of working with functions defined on $\mathbb{R}^{+}$, we could have worked with functions defined on any interval of the type $\left[t_{0}, \infty\right)$, obtaining a result completely similar. Also, many times, one is just interested in giving partial solutions; that is, solutions defined not on the whole interval $\left[t_{0}, \infty\right)$, but on some other interval $\left[t_{1}, \infty\right)$ with $t_{1} \geq t_{0}$.

Next, we just mention an easy result on the existence of solutions of the underlying differential equation, just to illustrate the type of functions $F$ that could generate a condition like (H1). This result is inspired from [7, Thm. 2] and $[8$, Thm. 1$]$.

Theorem 6. Let $F: \mathbb{R}^{+} \times X^{N} \rightarrow X$ be a continuous function mapping bounded sets into relatively compact ones, such that,

$$
\begin{array}{r}
\left\|F\left(t, y_{1}, \ldots, y_{N}\right)\right\|_{X} \leq \psi(t), \\
\forall t \in \mathbb{R}^{+}, \forall\left(y_{1}, \ldots, y_{N}\right) \in X^{N},
\end{array}
$$

where

$$
\psi: \mathbb{R}^{+} \longrightarrow \mathbb{R}^{+}, \text {satisfies } \int_{0}^{\infty} s \psi(s) d s<\infty
$$

Let also $\left\{\gamma_{j}\right\}_{j=1}^{N}$ be a set of continuous functions from $\mathbb{R}^{+}$to $\mathbb{R}^{+}$.

Then, for any $\omega \in \mathscr{C}^{2}\left(\mathbb{R}^{+}, X\right)$, (1) has a solution $y \in$ $\mathscr{C}^{2}\left(\mathbb{R}^{+}, X\right)$ with $y(t)-\omega(t) \rightarrow 0$ as $t \rightarrow \infty$.

Proof. Take $\omega \in \mathscr{C}^{2}\left(\mathbb{R}^{+}, X\right)$, consider the corresponding integral equation (2), and define

$$
g(t)=\int_{t}^{\infty} s \psi(s) d s, \quad t \geq 0
$$

Observe that, by (37), $g(t) \rightarrow 0$ as $t \rightarrow \infty$. This allows us to consider the set

$$
C=\left\{y \in \mathscr{C}\left(\mathbb{R}^{+}, X\right):\|y(t)-\omega(t)\|_{X} \leq g(t), t \geq 0\right\},
$$

which, adopting the notation used throughout the paper, gives by (36), for $y \in C$ and $t \geq 0$,

$$
\int_{t}^{\infty}\|(s-t) F(s, \Gamma(y)(s))\|_{X} d s \leq \int_{t}^{\infty}(s-t) \psi(s) d s \leq g(t) .
$$

With this, Theorem 3 applies (hypothesis $(\mathrm{H} 2)$ need not be verified by Remark 4 ) and then there exists $y \in \mathscr{C}\left(\mathbb{R}^{+}, X\right)$, solution of (2) with $y(t)-\omega(t) \rightarrow 0$ as $t \rightarrow \infty$. Finally, it is just an exercise to check that $y$ is twice continuously differentiable and that satisfies the differential equation (1).

\section{Conflict of Interests}

The authors declare that there is no conflict of interests regarding the publication of this paper.

\section{Acknowledgments}

Research partially supported by the Spanish (Grant MTM2011-25502) and regional Andalusian (Grants FQM210 and P09-FQM4468) Governments.

\section{References}

[1] F. V. Atkinson, "On second order nonlinear oscillation," Pacific Journal of Mathematics, vol. 5, pp. 643-647, 1955.

[2] A. Constantin, "On the existence of positive solutions of second order differential equations," Annali di Matematica Pura ed Applicata. Series IV, vol. 184, no. 2, pp. 131-138, 2005.

[3] S. G. Dubé and A. B. Mingarelli, "Note on a non-oscillation theorem of Atkinson," Electronic Journal of Differential Equations, vol. 2004, article 22, 6 pages, 2004, http://ejde.math.txstate.edu.

[4] M. Ehrnström, "Positive solutions for second-order nonlinear differential equations," Nonlinear Analysis: Theory, Methods \& Applications A, vol. 64, no. 7, pp. 1608-1620, 2006.

[5] M. Ehrnström, "Linear asymptotic behaviour of second order ordinary differential equations," Glasgow Mathematical Journal, vol. 49, no. 1, pp. 105-120, 2007.

[6] C. González and A. Jiménez-Melado, "Existence of monotonic asymptotically constant solutions for second order differential equations," Glasgow Mathematical Journal, vol. 49, no. 3, pp. 515-523, 2007.

[7] C. González and A. Jiménez-Melado, "Asymptotic behavior of solutions to an integral equation underlying a second-order differential equation," Nonlinear Analysis: Theory, Methods \& Applications A, vol. 70, no. 2, pp. 822-829, 2009.

[8] O. Lipovan, "On the asymptotic behaviour of the solutions to a class of second order nonlinear differential equations," Glasgow Mathematical Journal, vol. 45, no. 1, pp. 179-187, 2003.

[9] O. G. Mustafa and Y. V. Rogovchenko, "Global existence of solutions with prescribed asymptotic behavior for second-order nonlinear differential equations," Nonlinear Analysis: Theory, Methods \& Applications A, vol. 51, no. 2, pp. 339-368, 2002.

[10] E. Wahlén, "Positive solutions of second-order differential equations," Nonlinear Analysis: Theory, Methods \& Applications A, vol. 58, no. 3-4, pp. 359-366, 2004.

[11] G. E. Ladas and V. Lakshmikantham, Differential Equations in Abstract Spaces, vol. 85 of Mathematics in Science and Engineering, Academic Press, New York, NY, USA, 1972.

[12] E. Zeidler, Nonlinear Functional Analysis and Its Applications. I, Fixed-Point Theorems, Springer, New York, NY, USA, 1986, Translated from the German by P. R. Wadsack. 
[13] J. Dugundji, Topology, Allyn and Bacon, Boston, Mass, USA, 1966.

[14] S. Mazur, "Uber die kleinste konvexe Menge, die eine gegebene kompakte Menge enthalt," Studia Mathematica, vol. 2, pp. 7-9, 1930. 


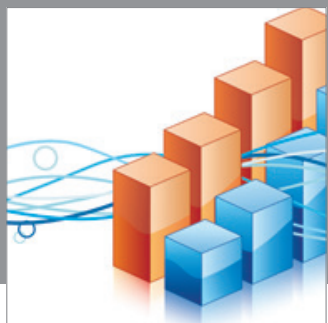

Advances in

Operations Research

mansans

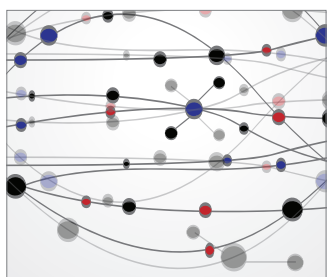

The Scientific World Journal
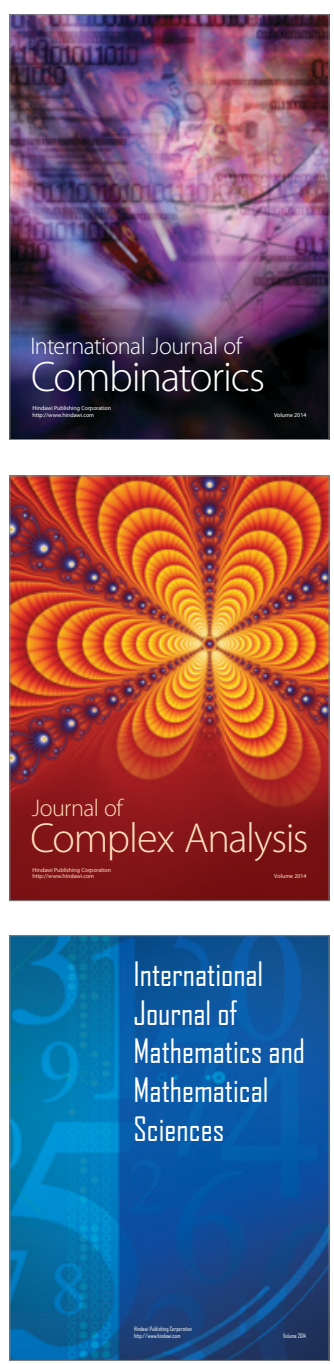
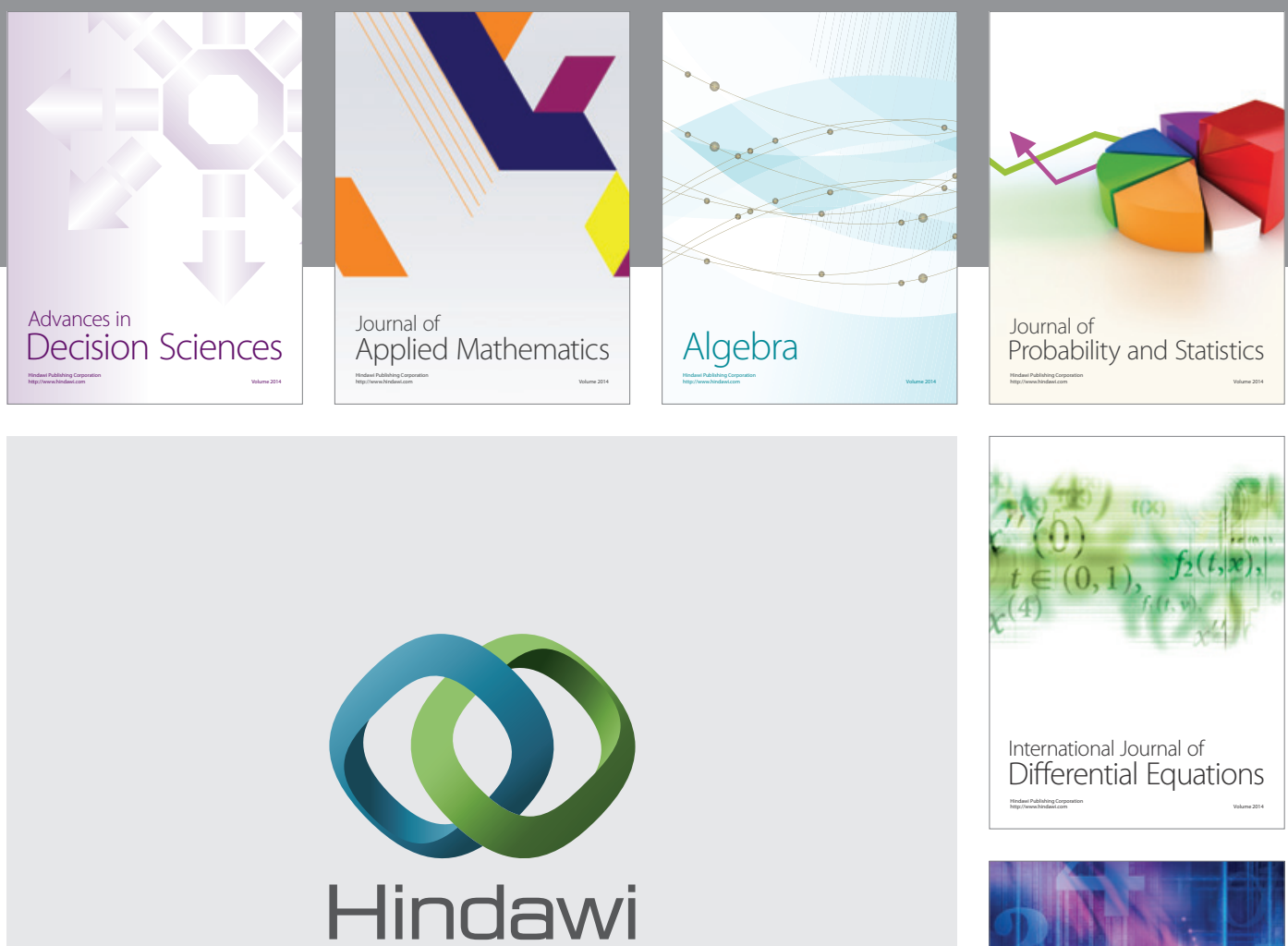

Submit your manuscripts at http://www.hindawi.com
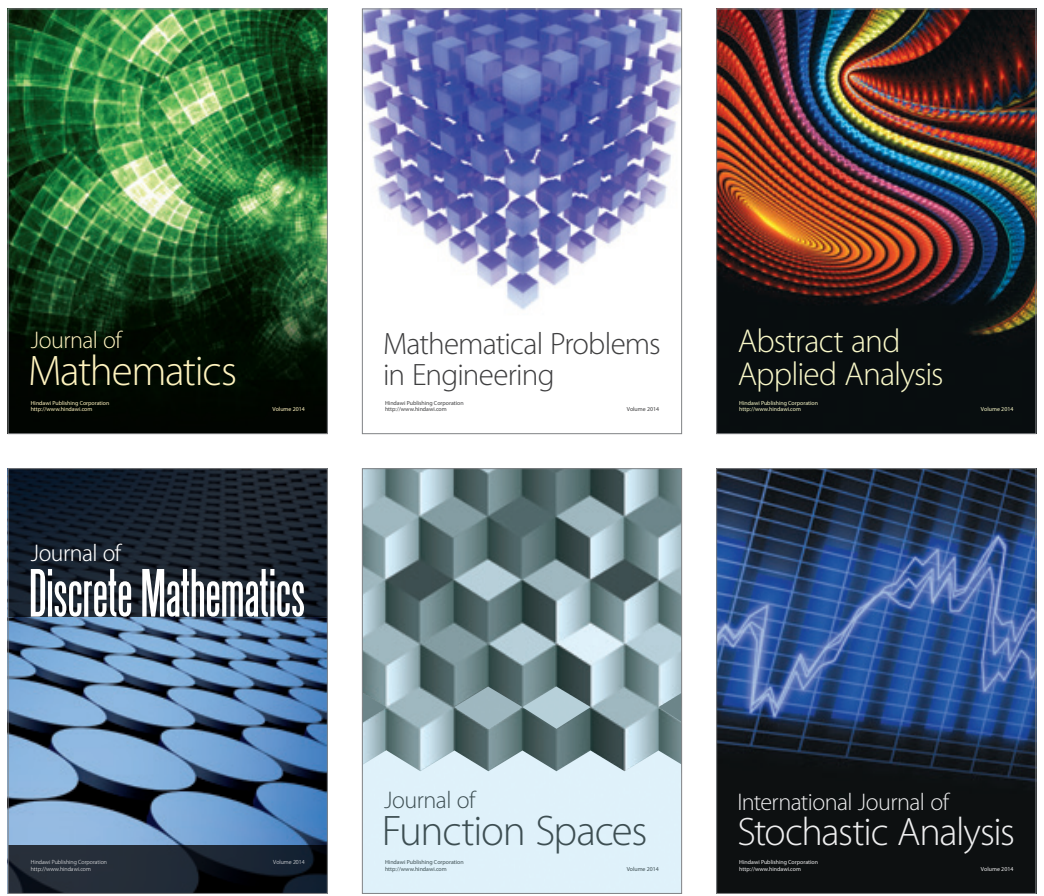

Journal of

Function Spaces

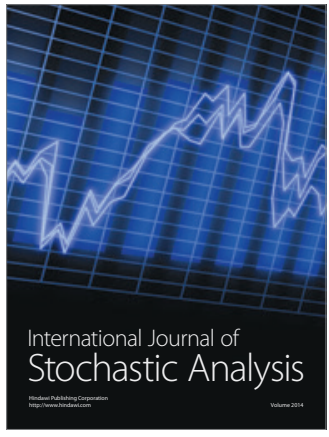

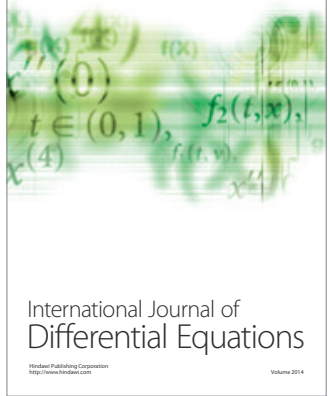
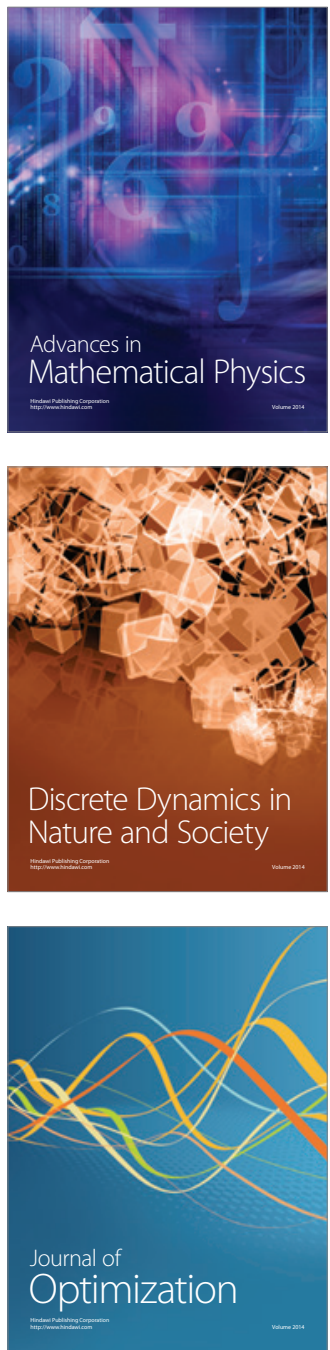